Rotational cooling of molecules using lamps

This article has been downloaded from IOPscience. Please scroll down to see the full text article.

2004 J. Phys. B: At. Mol. Opt. Phys. 374571

(http://iopscience.iop.org/0953-4075/37/22/015)

The Table of Contents and more related content is available

Download details:

IP Address: 130.225.29.254

The article was downloaded on 22/07/2009 at 16:39

Please note that terms and conditions apply. 


\title{
Rotational cooling of molecules using lamps
}

\author{
I S Vogelius ${ }^{1}$ L B Madsen ${ }^{1}$ and M Drewsen ${ }^{1,2}$ \\ ${ }^{1}$ Department of Physics and Astronomy, University of Aarhus, 8000 Århus C, Denmark \\ ${ }^{2}$ QUANTOP-Danish National Research Foundation Center for Quantum Optics, \\ Department of Physics and Astronomy, University of Aarhus, 8000 Århus C, Denmark
}

Received 31 August 2004, in final form 2 September 2004

Published 8 November 2004

Online at stacks.iop.org/JPhysB/37/4571

doi:10.1088/0953-4075/37/22/015

\begin{abstract}
We investigate theoretically the application of tailored incoherent far-infrared fields in combination with laser excitation of a single rovibrational transition for rotational cooling of translationally cold polar diatomic molecules. The cooling schemes are effective on a timescale shorter than typical unperturbed trapping times in ion traps and comparable to obtainable confinement times of neutral molecules.
\end{abstract}

\section{Introduction}

In recent years, the physics of cold molecules has become a very active research area (Doyle and Friedrich 1999, Williams and Julienne 2000, Egorov et al 2002, Bethlem et al 2002, Jochim et al 2003, Zwierlein et al 2003, Greiner et al 2003).

In this short paper we discuss the possibility of rotationally cool polar molecules by incoherent far-infrared radiation in combination with a single laser-excited rovibrational transition. Specifically, we consider molecules which are already translationally cold but where the rotational and vibrational degrees of freedom are in equilibrium with black-body radiation (BBR) at a temperature of $300 \mathrm{~K}$ (Mølhave and Drewsen 2000). For lighter molecules, the population will then be distributed over many rotational states, while only the vibrational ground state will be populated. The presented cooling schemes are, from an experimental point of view, significantly simpler than our previous proposed schemes which required two laser-induced transitions (Vogelius et al 2002). The incoherent field is tailored for optimum cooling into the rovibrational ground state under the constraints set by the spectral density profile of a mercury lamp (Kimmit et al 1996). The timescale for the cooling is shorter than the typical unperturbed trapping time in an ion trap (Mølhave and Drewsen 2000) and comparable to realistic trapping times for neutral molecules (van de Meerakker et al 2001).

\section{Cooling scheme}

Figure 1 shows laser excitations and subsequent spontaneous emission paths for our proposed rotational cooling schemes. With $(v, N)$ denoting the vibrational $v$ and rotational $N$ quantum 


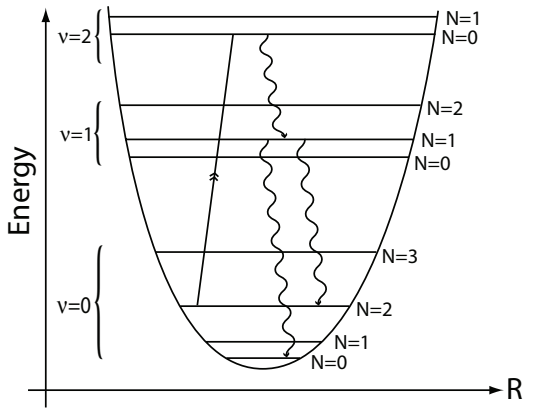

(a)

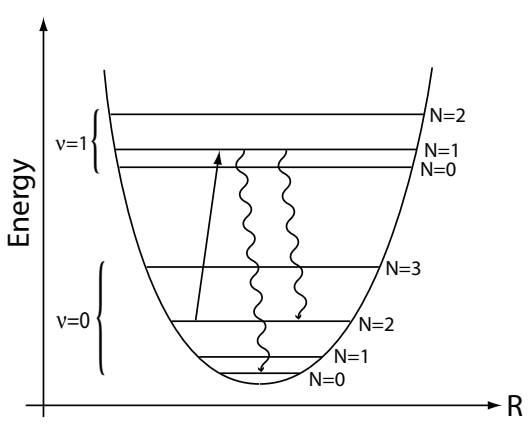

(b)

Figure 1. Sketch of potential energy curves and rovibrational energy levels showing laser driven transitions and spontaneous decay paths for the (a) Raman scheme and (b) direct scheme. Line with double-arrow: transition between rovibrational states driven by Raman pulses. Line with singlearrow: direct dipole laser excitations. Wiggly arrows: spontaneous decays. The internuclear distance is denoted by $R$.

numbers, the laser-induced transitions can either be Raman transitions via an excited electronic state between the levels $(0,2)$ and $(2,0)$ (a) or direct transitions between levels $(0,2)$ and $(1,1)$ (b). In both cases, the combined effect of laser excitations and subsequent spontaneous decay is to pump the molecules from the $(0,2)$ level into the $(0,0)$ ground state. In combination with BBR-induced transitions a significant enhancement in the population of the $(0,0)$ state can be obtained. At a specific temperature, the strength of the individual BBR-induced transitions is fixed, but by introducing additional incoherent far-infrared radiation derived from, e.g., a high-pressure mercury lamp, and by applying frequency filters to tailor the radiation intensity, specific rotational transition rates can be enhanced for improved cooling. One can, for instance, use a high-pass filter (Winnewisser et al 1999) such that the rate of the $(0,1) \leftrightarrow(0,2)$ cooling transition will be enhanced without effecting the rate of the $(0,0) \leftrightarrow(0,1)$ heating transition.

Though our schemes are widely applicable, we now focus on implementations in the particular case of $\mathrm{MgH}^{+}$which has been translationally cooled experimentally (Mølhave and Drewsen 2000). We showed previously that the laser intensities needed to saturate the Raman transition depicted in figure 1(a) are obtained by standard pulsed laser systems (Vogelius et al 2002). For the direct scheme (figure 1(b)), the infrared field $\left(\sim 5.9 \mu \mathrm{m}\right.$ for $\mathrm{MgH}^{+}$) could, e.g., be obtained by difference-frequency generation. Given the Einstein A-coefficient of $\sim 20 \mathrm{~s}^{-1}$ for the relevant transition, and assuming a maximum detuning of $1 \mathrm{GHz}$ due to fluctuations in the frequency of the laser light, a $1 \mu \mathrm{J}, 10 \mathrm{~ns}$ pulse focused to a realistic beam spot size of $1 \mathrm{~mm}^{2}$ will saturate the transition.

Turning to the lamp, the presence of this source may improve the cooling rate by speeding up the feeding of the $(0,2)$ pump state from higher-lying states. The transfer of population away from the pump state is, however, inevitable and it may thus be expected that a certain spectral density distribution of the incoherent radiation will cool optimally. We now investigate this hypothesis under realistic experimental constraints. The energy density of light in a standard mercury lamp at the wavelength of interest $(\sim 500 \mu \mathrm{m}$, corresponding to low-lying rotational transitions in $\mathrm{MgH}^{+}$) is similar to a $4000 \mathrm{~K} \mathrm{BBR}$ source (Kimmit et al 1996). Assuming unit magnification of the light source and a collection solid angle of $2 \pi$, which seems reasonable using a reflector and a large aperture molded lens (Nicolaisen 2003), the intensity of the lamp, in the important part of the spectrum at the position of the molecules, $I_{\text {lamp}}$, can exceed five times the intensity of the BBR, $I_{\mathrm{BBR}}$, at a temperature of $300 \mathrm{~K}$. 


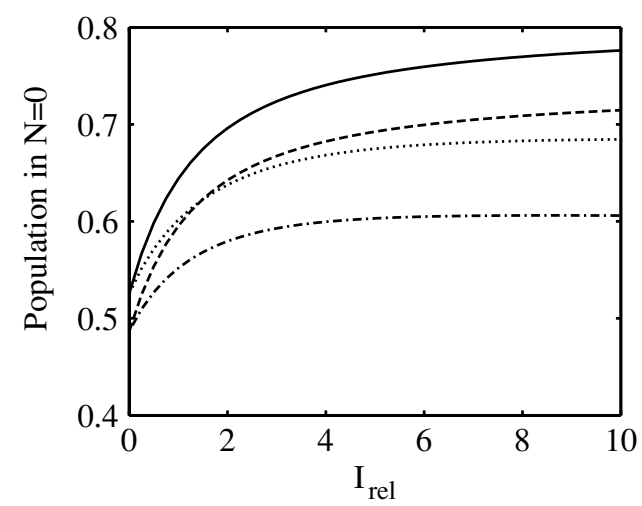

Figure 2. Ground state population of $\mathrm{MgH}^{+}$after $60 \mathrm{~s}$ of cooling versus relative intensity of incoherent radiation from a lamp. The schemes are direct scheme with optimal incoherent radiation distribution (solid) and direct scheme with 'quartz-filtered' distribution (dotted), Raman scheme with optimal incoherent radiation distribution (dashed) and Raman scheme with 'quartz-filtered' incoherent radiation distribution (dashed-dotted). See text for details. Note scale of the ordinate.

\section{Numerical simulations and conclusion}

As in previous works (Vogelius et al 2002, 2004), we model the cooling dynamics by rate equations including transitions induced by the incoherent radiation as well as by the laser(s). The molecular parameters are obtained by calculating Born-Oppenheimer potential energy curves and dipole moment functions using standard quantum chemistry codes (Frisch et al 1995) followed by the calculation of radial wavefunctions using the Numerov method (LeRoy 2002). The nuclear wavefunction and the electronic dipole moment function determine the Einstein coefficients. All simulations are made with an initial $300 \mathrm{~K}$ Boltzmann distribution over molecular energy levels and a pulsed laser system with a repetition rate of $100 \mathrm{~Hz}$ saturating the driven transition during each pulse.

We maximize the final population in the ground state as a function of the incoherent radiation density at the individual rotational transition frequencies for specific cooling times by the downhill simplex method (Lagarias et al 1998). We find that the density distribution should be maximal for the $(0,1) \leftrightarrow(0,2),(0,2) \leftrightarrow(0,3)$, and $(0,3) \leftrightarrow(0,4)$ transitions and zero otherwise, and that the introduction of a time-dependent field only improves the cooling efficiency marginally. This simple shape may be understood by noting that for a molecule subject to BBR, stimulated processes dominate at low frequencies while spontaneous emission do so at high frequencies. As a result, the highest populated state, $(\nu, N)_{\max } \mathrm{BBR}$ is that where spontaneous and stimulated transition rates are balanced $\left((v, N)_{\max }\right.$ BBR $=(0,4)$ for $\left.\mathrm{MgH}^{+}\right)$. The BBR density is low and transition rates are small for rotational states lying below $(v, N)_{\max }$ BBR. Introducing additional incoherent radiation to drive transitions between these low-lying states will accelerate the process of refilling the $(0,2)$ pump state, and thereby increase the cooling rate. Radiation which couples states above the peak in the $300 \mathrm{~K} \mathrm{BBR}$ population distribution would heat the distribution as would any radiation in addition to BBR at the $(0,0) \leftrightarrow(0,1)$ transition frequency.

Figure 2 shows the cooling efficiency as a function of $I_{\text {rel }}=\frac{I_{\text {lamp }}}{I_{\mathrm{BBR}}}$. The influence of the incoherent field on the cooling process is seen to increase significantly up to $I_{\text {rel }} \approx 5$ from where only minor improvements may be obtained. This intensity level coincides well with the estimate obtainable with a mercury lamp. 
The schemes discussed here can be extended to ${ }^{2} \Sigma,{ }^{3} \Sigma$ and ${ }^{2} \Pi$ electronic ground states without losing their experimental feasibility, and should therefore be applicable to almost any diatomic molecule with rotational and vibrational transition rates comparable to those of $\mathrm{MgH}^{+}$or faster (Vogelius et al 2004).

In conclusion, we have demonstrated that polar molecules trapped in a room temperature environment can be cooled rotationally by the combination of a lamp inducing selected rotational transitions and a laser driving a single rovibrational transition. Under realistic constraints we have shown that there exists a specific tailored incoherent frequency distribution which optimizes the cooling process. From an experimental view-point, the considered schemes are very simple, and hence attractive for sympathetically cooled target molecular ions, and potentially also for trapped neutral molecules and molecular ions in storage rings.

\section{Acknowledgments}

LBM is supported by the Danish Natural Science Research Council (grant no 21-03-0163). MD acknowledges financial support from the Danish National Research Foundation through the Quantum Optics Center QUANTOP.

\section{References}

Bethlem H, Crompvoets F, Jongma R, van de Meerakker S and Meijer G 2002 Phys. Rev. A 65053416

Doyle J M and Friedrich B 1999 Nature 401749

Egorov D, Lahaye T, Schöllkopf W, Friedrich B and Doyle J M 2002 Phys. Rev. A 66043401

Frisch M et al 1995 Gaussian 94, revision e.1 Technical Report (Pittsburgh, PA: Gaussian Inc.)

Greiner M, Regal C A and Jin D S 2003 Nature 426537

Jochim S, Bartenstein M, Altmeyer A, Hendl G, Riedl S, Chin C, Denschlag J H and Grimm R 2003 Science 302 2101

Kimmit M, Walsh J, Platt C, Miller K and Jensen M 1996 Infrared Phys. Techn. 37471

Lagarias J C, Reeds J A, Wright M H and Wright P E 1998 SIAM J. Optim. 9112

LeRoy R J 2002 Level 7.5: A Computer Program for Solving the Radial Schrödinger Equation for Bound and Quasibound Levels The source code and manual for this program may be obtained from the 'Computer Programs' link on the www site http://leroy.uwaterloo.ca

Mølhave K and Drewsen M 2000 Phys. Rev. A 62011401 (R)

Nicolaisen F M 2003 Private communication

van de Meerakker S Y, Jongma R T, Bethlem H L and Meijer G 2001 Phys. Rev. A 64041401

Vogelius I S, Madsen L B and Drewsen M 2002 Phys. Rev. Lett. 89173003

Vogelius I S, Madsen L B and Drewsen M 2004 Phys. Rev. A at press

Williams C and Julienne P 2000 Science 287986

Winnewisser C, Lewen F, Weinzierl J and Helm H 1999 Appl. Opt. 383961

Zwierlein M W, Stan C A, Schunck C H, Raupach S M F, Gupta S, Hadzibabic Z and Ketterle W 2003 Phys. Rev. Lett. 91250401 